\title{
Comparative effects of nebivolol and carvedilol on left ventricular diastolic function in older heart failure patients with preserved ejection fraction: study protocol for a randomized controlled trial
}

Kyungil Park and Tae-Ho Park ${ }^{*}$

\begin{abstract}
Background: Heart failure (HF) is a common and disabling condition in older people. Randomized clinical trials and meta-analyses have clearly demonstrated that the long-term use of $\beta$-Blockers improves the outcome of patients with HF. However, limited data are available on the treatment of older HF patients with preserved ejection fraction (EF). No study has specifically compared the relative effectiveness of carvedilol and nebivolol in treating HF in older patients with preserved EF.

Method/design: This trial is a prospective, randomized, open-label, single-centre, active controlled study designed to investigate the effects of nebivolol and carvedilol on diastolic function of the left ventricle (LV) in older HF patients with preserved EF.

We will test the hypothesis that nebivolol improves LV diastolic function to a greater extent than carvedilol in patients over 70 years of age. The study population includes 62 older patients newly diagnosed with HF. Patients will be included in the study if they have a LVEF $\geq 40 \%$, New York Heart Association (NYHA) functional classes I, II or III status, and have been clinically stable without hospital admission for HF in the preceding 3 months. Eligible patients will be randomly assigned, in a 1:1 ratio, to receive a loading and maintenance dose of either nebivolol or carvedilol. Echocardiographic evaluations will be performed at baseline, 6, and 12 months after therapy. Clinical assessment and laboratory tests are to be performed at fixed times.
\end{abstract}

Discussion: This trial is a single-center study that aims to evaluate the impact of nebivolol on LV diastolic function. The results of the study will provide information about the optimal choice of a $\beta$-Blocker in the management of patients after diagnosis of HF with preserved EF. The results will be available by 2017.

Trial registration: ClinicalTrials.gov Identifier: NCT02619526, registered on 25 November 2015.

Keywords: Nebivolol, Carvedilol, Heart failure

\footnotetext{
* Correspondence: thpark65@dau.ac.kr

Division of Cardiology, Department of Internal Medicine, Dong-A University

College of Medicine, 1 Dongdae-sin-dong 3-ga, Seo-gu, Busan 602-715,

South Korea
} 


\section{Background}

Heart failure (HF) is a common disease in older people, and has a pronounced negative impact on quality of life and functional status $[1,2]$. The use of $\beta$-Blockers has been proven to improve left ventricular (LV) systolic function and the prognoses of patients with HF in randomized controlled trials [3-6]. Thus, $\beta$-Blockers are considered a cornerstone in the pharmacological treatment of HF. However, data have shown that the median age of presentation of new $\mathrm{HF}$ cases is older than 75 years $[2,7,8]$. For patients with $\mathrm{HF}$ aged 70 and over the evidence for the efficacy of $\beta$-Blockers is limited. Only the SENIORS (Study of Effects of Nebivolol Intervention on Outcomes and Rehospitalization in Seniors With Heart Failure) trial has investigated the effect of nebivolol in HF patients over the age of 70 years. Those results demonstrated a significant reduction in the risk of death and cardiovascular-related hospitalization in patients given $\beta$-Blockers as compared with a placebo [6]. In a subanalysis of the SENIORS trial, the effect of nebivolol in older patients with HF was shown to be similar in those with preserved and impaired ejection fraction (EF) [9].

However, no study has specifically compared the relative effectiveness of nebivolol and carvedilol in treating HF in older patients with preserved LV function. This open-label study will compare the effects of long-term treatment with nebivolol versus carvedilol on LV diastolic function in older patients with HF.

\section{Trial objectives}

The primary question to be tested is whether nebivolol improves LV diastolic function as measured by echocardiography in older patients with preserved EF during a follow-up period of 1 year, compared with the usual dose of carvedilol in the control group.

The secondary objective of the study is to assess the effect of the two $\beta$-Blockers on clinical outcomes. Clinical events are defined as symptom severity (New York Heart Association (NYHA) classification) and hospitalization due to $\mathrm{HF}$.

\section{Methods/design}

\section{Ethics}

This study follows the principles set forth in the Helsinki Declaration, meaning that all patients sign a written informed consent stating that participation is voluntary and that participation can be withdrawn at any time, without any negative consequences concerning their current or future medical treatment. The study protocol (version \#1, 7 October 2015), which follows the Standard Protocol Items: Recommendations for Interventional Trials (SPIRIT) guidelines (see Additional file 1), has been approved by the Institutional Review Board of Dong-A University Hospital.

\section{Patient population}

This study will take place at Dong-A University Hospital. Patients will be eligible for the study if they have a left ventricular ejection fraction (LVEF) $\geq 40 \%$, NYHA functional classes I, II or III status, and have been clinically stable without hospital admission for HF in the preceding 3 months. Men and women over 70 years of age are eligible (Table 1). In the present study, preserved LVEF is defined as an EF $\geq 40$ \% using a modified Simpson's rule [10]. Pharmacological therapy other than that with the study drug, including ACE and statin use, is allowed at the discretion of the attending physician. Patients who have contraindications to the study drug or major concomitant diseases are to be excluded (Table 1).

\section{Study design}

This study is a prospective, randomized, open-label, single-centre, active controlled study with two parallel study groups. The choice of a prospective and openlabel study was felt to be a reasonable reflection of clinical practice in only one center with a small sample. The randomized design was adopted to minimize bias in this single-center study. The dose of nebivolol was chosen on the basis of the SENIORS study [6]. The control arm will receive carvedilol because it is the $\beta$-Blocker that is

Table 1 Eligibility criteria

\section{Inclusion criteria}

Subjects $>70$ years of age

Either gender

First diagnosis of heart failure defined as:

a) Mild or moderate symptomatic heart failure (NYHA calss I to III)

b) An echocardiographic left ventricular ejection fraction $\geq 40 \%$

Patients who provide written informed consent

\section{Exclusion criteria}

History and/or clinical documentation of pulmonary embolism Severe heart failure (NYHA IV or need for inotropic support) Primary valvular heart disease

Pericardial disease

Severe obstructive lung disease

Primary pulmonary hypertension

Occupational lung disease

Asthma

Severe renal failure (serum creatinine $>2.0 \mathrm{mg} / \mathrm{dL}$ )

Significant peripheral vascular disease

Severe bradycardia (heart rate $<50$ beats/minutes)

Second or third-degree atrio-ventricular block

Atrial fibrillation

Life expectancy $<1$ year

Concern for inability of the patient to comply with study procedures and/or follow up

Any condition which in the opinion of the Investigator would make it unsafe or unsuitable for the patient to participate in this study Involvement in the planning and/or conduct of the study

Participation in another clinical study with an investigational product during the preceding 30 days

Unable to give informed consent 
indicated for the broadest population and is the most frequently used $\beta$-Blocker worldwide. A treatment duration of a minimum of 12 months was selected as a result of the SENIORS trial. The overall study design is depicted in Fig. 1.

\section{Randomization}

All new patients admitted to the Dong-A University Cardiology Department will be screened for participation eligibility in the study by the principal investigator. Randomization following initial echocardiographic testing can be performed immediately after enrollment for patients who fulfill all the eligibility requirements. Patients will be randomly allocated to one of two conditions: (1) nebivolol treatment (10 mg once daily) or (2) carvedilol treatment ( $25 \mathrm{mg}$ twice daily). Enrollment is expected to continue for approximately 12 months. Random treatment assignments will be generated using Excel spreadsheet software (Microsoft Corporation, Redmont, DC, USA). Eligible patients will be randomly assigned in a 1:1 ratio to receive either nebivolol or carvedilol. Randomization will be performed by an individual not involved in the study and will be kept concealed. Following randomization, the first dose of study medication will be administered to the patient as soon as possible. Potential attrition bias will be mitigated by only including patients who are motivated to recieve treatment. We will take efforts to minimize dropout and any potential biases attributable to dropout will be explored statistically. If a participant wishes to withdraw from the study, the reason for withdrawal will be documented in the participant's records for the subsequent analysis in the interpretation of the results.

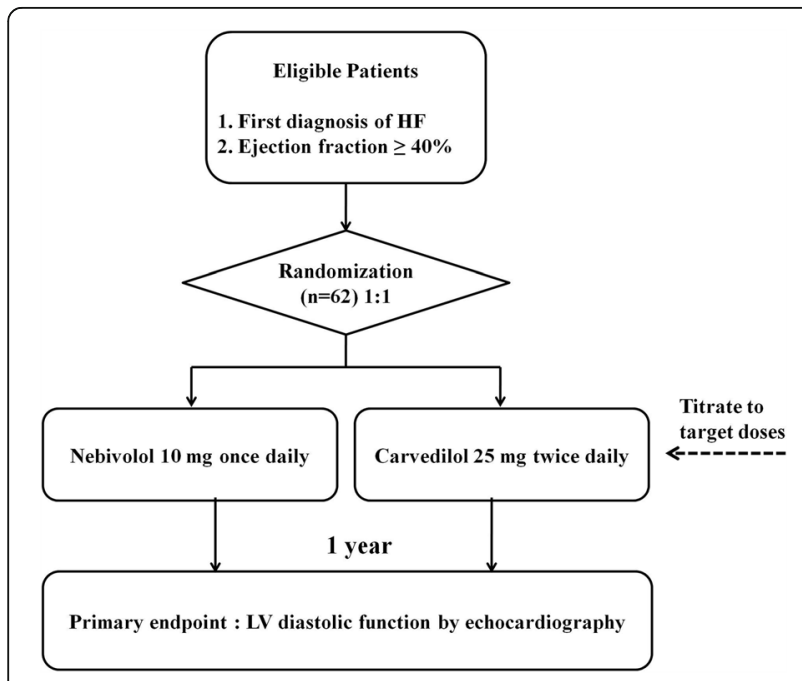

Fig. 1 Overall algorithm of the study

\section{Outcome measurements}

The primary efficacy variables are mitral inflow velocities (E and A waves), deceleration time of the $\mathrm{E}$ wave (DT), isovolumetric relaxation time (IVRT), mitral annular velocities (early diastolic velocity of mitral annulus (Ea) and late diastolic velocity of mitral annulus (Aa) waves), left atrial (LA) volume, and LA strain (global systolic and diastolic LA strain), which will be measured via echocardiographic evaluation after 12 months of treatment. Mitral E/A and E/Ea ratios will be calculated. Parameters from Doppler analysis, M-mode echocardiography, and 2D-transthoracic echocardiography will be used. All echocardiographic images will be interpreted by an observer who is blinded to clinical data.

Secondary outcomes include occurrence of clinical events. Symptom severity (NYHA classification) and hospitalization will be evaluated at baseline and at maintenance follow-up visits. Vital signs, including heart rate and blood pressure, will be measured at every visit. Any complications, discontinuations, and deaths occurring during the study period will be noted. Each patient's medical history and the results of a clinical examination will be recorded at baseline by the attending physician.

\section{Intervention and comparator descriptions}

Patients will be followed with weekly visits during the up-titration phase. For nebivolol, the initial dose will be $1.25 \mathrm{mg}$ once daily. If tolerated, it will be increased to $2.5 \mathrm{mg}$ once daily by the end of week $1,5 \mathrm{mg}$ once daily by week 2 (visit 1 ), and $10 \mathrm{mg}$ once daily by week 4 (visit 2). For carvedilol, the initial dose will be half of a 3.125mg tablet twice daily. If tolerated, it will be increased to $6.25 \mathrm{mg}$ twice daily by the end of week $1,12.5 \mathrm{mg}$ twice daily by week 2 (visit 1), and $25 \mathrm{mg}$ twice daily by week 4 (visit 2). If side effects attributable to the study medications occur, up-titration will be delayed, the dose will be decreased, or the administration of the drug will be temporarily discontinued. If up-titration is not clinically feasible, either because of hypotension or bradycardia, the previous dose will be administered subsequently as the maximal tolerable dose. This process is illustrated in Fig. 2.

\section{Follow-up protocol}

On achievement of the target dose or the maximum tolerable dose, patients will be followed up clinically every month for the first 3 months, then every 3 months until the end of the study at 12 months. At each visit, patients will undergo a complete physical examination, medical history-taking, and assessment of drug compliance. Investigators will evaluate all clinical and laboratory adverse events at each visit. NYHA functional class and predefined clinical events will be recorded at each 


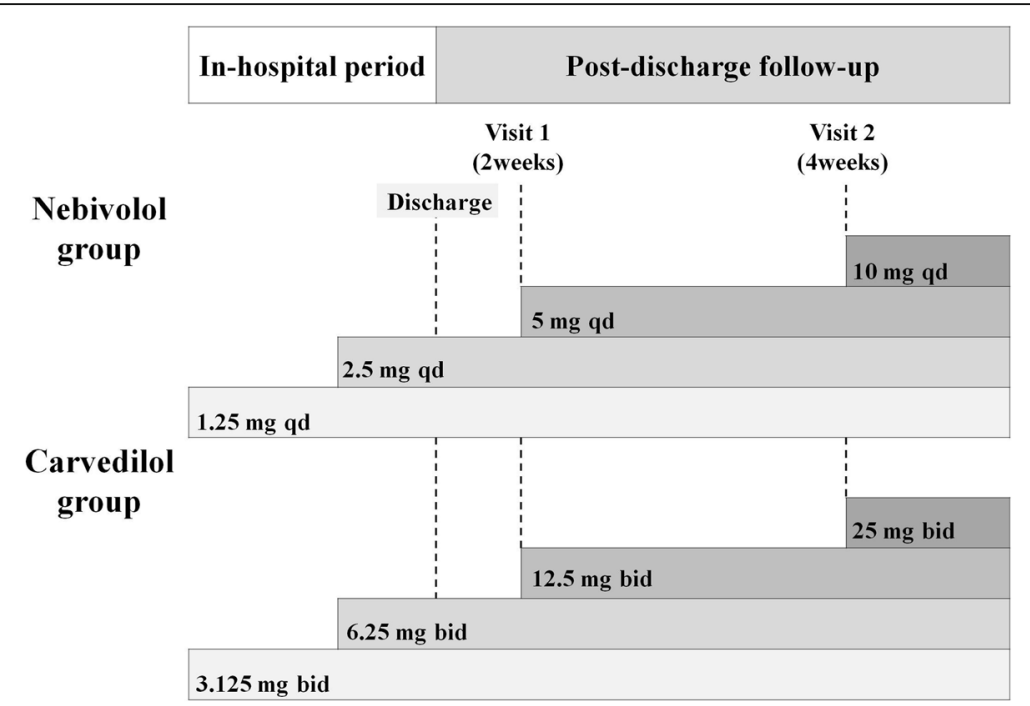

Fig. 2 Titration scheme of the study drug

clinical visit. Echocardiographic examinations will be performed at 6 and 12 months after discharge.

\section{Adverse effects}

Analysis of safety-related data will be performed with respect to frequency of serious adverse events (SAE), stratified by causality and intensity of morbidity in both treatment groups. Patients will be interviewed at each visit about the occurrence of any adverse events, including the time of onset, duration, and severity; all information will be recorded on a Case Report Form. The causal relation to the study drug and the intensity of adverse events will be evaluated by the investigators. SAE must be reported to the Institutional Review Board and study sponsor by the principal investigator within $24 \mathrm{~h}$ after the SAE becomes known.

\section{Withdrawals}

Patients are free to withdraw from participation in the trial at their own request at any time and without giving reasons for their decision. Moreover, the primary investigator can withdraw study patients if continuation of the trial would be detrimental to the patient's wellbeing. Withdrawals will be documented in the Case Report Form and in the patient's medical records and all ongoing SAE will be followed up.

\section{Sample size}

Calculation of sample size was done based on the primary outcomes and the primary analysis for the intention-to-treat population. The sample size was calculated using the study's primary objective, to detect a difference in E/E' of 5.0 between nebivolol and carvedilol treatment with a power of $90 \%$. On the basis of previous reports, we assume the standard deviation of E/E' to be approximately 5.0 in both treatment groups $[11,12]$. We adjusted the sample size for an estimated follow-up loss rate of $30 \%$, a two-sided level of significance $a=5 \%$ and a power of $1-\beta=90 \%$. Thirty-one patients in each group are required to detect statistical differences with a twosided Student's $t$ test given these parameters. Therefore, a total of 62 patients will be included in the analysis. The statistical analyses will be performed on the full dataset, which will include data from all randomized patients receiving at least one dose of study medication. Patients who are judged to be ineligible after study registration will be excluded from the analysis. Given the sample size, this study is likely to be underpowered in terms of assessing secondary outcomes.

\section{Data collection, storage, and security}

All paper files are to be stored in locked file cabinets, and electronic files are to be stored in passwordprotected, encrypted files. Both paper and electronic files will be accessed only by members of the research team. The final dataset will reside with the principal investigator.

\section{Statistical analyses}

The intent-to-treat (ITT) analysis set will be used for efficacy analyses. All patients who have begun treatment will be included irrespective of their protocol adherence and continued participation in the study. Patients will be analyzed according to their randomized study medication irrespective of whether a SAE occurred before or following discontinuation of study medication. For baseline characteristics, continuous variables will be assessed using the Student's $t$ test and discrete variables will be 
compared using the chi-square test. The primary outcome will be compared using two-sample $t$ tests. The two-sided null hypothesis for the primary outcome measure states that nebivolol and carvedilol lead to the same change in ventricular diastolic function during the 12 months after diagnosis. This null hypothesis will be tested by regression analysis adjusted for baseline values. Assessments for other echocardiographic indices will be compared between treatment groups after 12 months of follow-up using two-sample $t$ tests. Analyses of changes will be performed using data from all patients for whom baseline and 12-month follow-up data are available. Event-free cumulative survival rates will be plotted using the Kaplan-Meier method and comparisons will be made between patients given nebivolol and carvedilol using the log-rank test. A Cox proportional hazards model, with the use of forward selection based on the likelihood ratio test, will be implemented for multivariate analysis to determine which prognostic factors identified in the univariate analysis, including mitral inflow velocities, DT, IVRT, mitral annular velocities, LA volume, and LA strain were significantly related to clinical events in the follow-up period.

A safety analysis dataset will be used to assess safety and tolerability variables. All patients who received at least one dose of nebivolol or carvedilol, and for whom post-dose data are available, will be included in the safety population. Patients will be censored 7 days after their last dose of study medication. A value of $p<0.05$ will be considered statistically significant.

Potential attrition bias will be mitigated by conducting dropout analysis and intention-to-treat analysis. Given that dropouts are expected, multiple imputation, based on regression methods, will be performed to complete the data. A fully specified statistical analysis plan will be written before unmasking of the data.

The report presenting the primary findings from this study will follow the Consolidated Standards of Reporting Trials (CONSORT) 2010 guidelines. Study results will be disseminated to researchers and clinicians via publications and conference presentations. Authorship of published papers will follow established guidelines for defining the level of contribution that warrants authorship.

\section{Discussion}

Aging represents both a convergence of declining cardioprotective systems and increasing disease processes that are fertile ground for the development of HF [2]. With $50 \%$ of all HF diagnoses and $90 \%$ of all HF deaths occurring in the segment of the population over age $70, \mathrm{HF}$ is largely a disease of older people. In addition, the greatest proportion of older HF patients have preserved systolic function [1].
Patients with HF can be divided into two different groups based on EF: HF with preserved or reduced EF [13]. The two are similar in terms of prevalence and mortality rate [14, 15]. However, current HF guidelines do not address the use of $\beta$-Blockers in patients with preserved EF [16] because no effective treatment has been identified in randomized clinical trials.

$\beta$-Blockers are thought to potentially improve diastolic filling by a negative chronotropic effect [17]. Some studies support the use of $\beta$-Blockers in patients with preserved EF $[18,19]$. Notably, recent data suggest that the effect of the $\beta$-Blocker nebivolol is similar in HF patients with preserved and impaired EF [9]. Increased nitric oxide release induced by nebivolol [20] may encourage early LV relaxation [21].

Randomized clinical trials and meta-analyses have shown that long-term use of $\beta$-Blockers improves LV systolic function and clinical outcomes of HF patients with reduced EF. The average age of subjects in these placebo-controlled mortality and morbidity studies of $\beta$-Blockers in HF has been below 70 years [3-5]. Several $\beta$-Blockers are available, but only four, carvedilol, metoprolol, bisoprolol, and nebivolol, have been successfully tested in HF and are used in treatment of the condition [22, 23]. However, comparison studies on the efficiency of different $\beta$-Blockers in the treatment of HF are limited [23, 24]. At present, little data are available on the comparative effectiveness of carvedilol and nebivolol for HF. A recent study found that carvedilol and nebivolol appear to similarly improve LV diastolic functions in nonischemic HF patients [25]. However, that study did not involve an HF population with preserved EF. Thus, the current trial will be the first head-to-head comparison of the diastolic effects of nebivolol and carvedilol in HF patients with preserved EF.

With regard to possible risks of bias, we anticipate that the risk of selection bias is low, due to the use of adequate methods for sequence generation and allocation sequence concealment. Regarding performance, participants might be influenced by knowledge of group assignment; however, the possibility that unblinding to group assignment may result in an overestimation or an underestimation of intervention effects is low. In addition, as reported above, the risk of attrition bias due to missing data has been adequately prevented and will be treated.

\section{Trial status}

Recruitment is ongoing at the time of manuscript submission.

\section{Additional file}

Additional file 1: Appendix A. SPIRIT 2013 checklist: recommended items to address in a clinical trial protocol and related documents*. Appendix B. World Health Organization Trial Registration Dataset. Appendix C. Informed consent (only for Korean participants. (DOC $182 \mathrm{~kb}$ ) 


\section{Abbreviations}

ACE: Angiotensin-converting enzyme; EF: Ejection fraction; HF: Heart failure; LV: Left ventricle; NYHA: New York Heart Association; SENIOR: Study of the Effects of Nebivolol Intervention on Outcomes and Rehospitalization in Seniors with Heart Failure

\section{Acknowledgements}

This study was financially supported by Menarini Korea, Seoul, Korea. As this is an investigator-initiated trial, the authors designed and conducted the study. Data collection is managed by an independent clinical research center. The funding body does not interfere in the analysis and interpretation of the data. We would like to thank all members of the present study group for their ideas, suggestions, participation, and support.

\section{Authors' contributions}

THP is responsible for general project coordination, study design, work data interpretation, and critical manuscript revision. KP is responsible for study concept, study sample size calculation, critical manuscript revision, and for all other aspects of the study. Both authors have read and approved the final manuscript.

\section{Competing interests}

The authors declare that they have no competing interests.

\section{Received: 24 December 2015 Accepted: 13 October 2016}

\section{Published online: 03 November 2016}

\section{References}

1. Hogg K, Swedberg K, McMurray J. Heart failure with preserved left ventricular systolic function. Epidemiology, clinical characteristics, and prognosis. J Am Coll Cardiol. 2004:43:317-27.

2. Strait JB, Lakatta EG. Aging-associated cardiovascular changes and their relationship to heart failure. Heart Fail Clin. 2012;8:143-64.

3. Packer M, Bristow MR, Cohn JN, Colucci WS, Fowler MB, Gilbert EM, Shusterman $\mathrm{NH}$. The effect of carvedilol on morbidity and mortality in patients with chronic heart failure. U.S. Carvedilol Heart Failure Study Group. N Engl J Med. 1996:334:1349-55.

4. CIBIS-II Investigators and Committees. The Cardiac Insufficiency Bisoprolol Study II (CIBIS-II): a randomised trial. Lancet. 1999;353:9-13.

5. MERIT-HF Study Group. Effect of metoprolol CR/XL in chronic heart failure: Metoprolol CR/XL Randomised Intervention Trial in Congestive Heart Failure (MERIT-HF). Lancet. 1999;353:2001-7.

6. Flather MD, Shibata MC, Coats AJ, Van Veldhuisen DJ, Parkhomenko A, Borbola J, Cohen-Solal A, Dumitrascu D, Ferrari R, Lechat P, Soler-Soler J, Tavazzi L, Spinarova L, Toman J, Böhm M, Anker SD, Thompson SG, PooleWilson PA, SENIORS Investigators. Randomized trial to determine the effect of nebivolol on mortality and cardiovascular hospital admission in elderly patients with heart failure (SENIORS). Eur Heart J. 2005:26:215-25.

7. Bui AL, Horwich TB, Fonarow GC. Epidemiology and risk profile of heart failure. Nat Rev Cardiol. 2011:8:30-41.

8. Chun S, Tu JV, Wijeysundera HC, Austin PC, Wang X, Levy D, Lee DS. Lifetime analysis of hospitalizations and survival of patients newly admitted with heart failure. Circ Heart Fail. 2012:5:414-21.

9. van Veldhuisen DJ, Cohen-Solal A, Böhm M, Anker SD, Babalis D, Roughton M, Coats AJ, Poole-Wilson PA, Flather MD, SENIORS Investigators. Betablockade with nebivolol in elderly heart failure patients with impaired and preserved left ventricular ejection fraction: data from SENIORS (Study of Effects of Nebivolol Intervention on Outcomes and Rehospitalization in Seniors With Heart Failure). J Am Coll Cardiol. 2009:53:2150-8.

10. Lang RM, Bierig M, Devereux RB, Flachskampf FA, Foster E, Pellikka PA, Picard MH, Roman MJ, Seward J, Shanewise JS, Solomon SD, Spencer KT, Sutton MS, Stewart WJ. American Society of Echocardiography's Guidelines and Standards Committee: European Association of Echocardiography. J Am Soc Echocardiogr. 2005;18:1440-63.

11. Kasner M, Westermann D, Steendijk P, Gaub R, Wilkenshoff U, Weitmann K, Hoffmann W, Poller W, Schultheiss HP, Pauschinger M, Tschöpe C. Utility of Doppler echocardiography and tissue Doppler imaging in the estimation of diastolic function in heart failure with normal ejection fraction: a comparative Doppler-conductance catheterization study. Circulation. 2007;116:637-47.

12. Guazzi M, Vicenzi M, Arena R, Guazzi MD. PDE5 inhibition with sildenafil improves left ventricular diastolic function, cardiac geometry, and clinical status in patients with stable systolic heart failure: results of a 1-year, prospective, randomized, placebo-controlled study. Circ Heart Fail. 2011:4:8-17.

13. Lee DS, Gona P, Vasan RS, Larson MG, Benjamin EJ, Wang TJ, Tu JV, Levy D. Relation of disease pathogenesis and risk factors to heart failure with preserved or reduced ejection fraction: insights from the Framingham Heart Study of the National Heart, Lung, and Blood Institute. Circulation. 2009;119:3070-7.

14. Steinberg BA, Zhao X, Heidenreich PA, Peterson ED, Bhatt DL, Cannon CP, Hernandez AF, Fonarow GC, Get With The Guidelines Scientific Advisory Committee and Investigators. Trends in patients hospitalized with heart failure and preserved left ventricular ejection fraction: prevalence, therapies, and outcomes. Circulation. 2012:126:65-75.

15. Brouwers FP, de Boer RA, van der Harst P, Voors AA, Gansevoort RT, Bakker SJ, Hillege HL, van Veldhuisen DJ, van Gilst WH. Incidence and epidemiology of new onset heart failure with preserved vs. reduced ejection fraction in a communitybased cohort: 11-year follow-up of PREVEND. Eur Heart J. 2013:34:1424-31.

16. Yancy CW, Jessup M, Bozkurt B, Butler J, Casey Jr DE, Drazner MH, Fonarow GC, Geraci SA, Horwich T, Januzzi JL, Johnson MR, Kasper EK, Levy WC, Masoudi FA, McBride PE, McMurray JJ, Mitchell JE, Peterson PN, Riegel B, Sam F, Stevenson LW, Tang WH, Tsai EJ, Wilkoff BL, American College of Cardiology Foundation/ American Heart Association Task Force on Practice Guidelines. 2013 ACCF/AHA guideline for the management of heart failure: a report of the American College of Cardiology Foundation/American Heart Association Task Force on Practice Guidelines. J Am Coll Cardiol. 2013;62:e147-239.

17. Ha JW, Oh JK. Therapeutic strategies for diastolic dysfunction: a clinical perspective. J Cardiovasc Ultrasound. 2009;17:86-95.

18. Lund $\mathrm{LH}$, Benson L, Dahlström U, Edner M, Friberg L. Association between use of $\beta$-blockers and outcomes in patients with heart failure and preserved ejection fraction. JAMA. 2014;312:2008-18.

19. Dobre D, van Veldhuisen DJ, DeJongste MJ, Lucas C, Cleuren G, Sanderman R, Ranchor AV, Haaijer-Ruskamp FM. Prescription of beta-blockers in patients with advanced heart failure and preserved left ventricular ejection fraction. Clinical implications and survival. Eur J Heart Fail. 2007;9:280-6.

20. Zanchetti A. Clinical pharmacodynamics of nebivolol: new evidence of nitric-oxide mediated vasodilating activity and peculiar haemodynamic properties in hypertensive patients. Blood Press. 2004;13:18-33.

21. Paulus WJ, Shah AM. NO and cardiac diastolic function. Cardiovasc Res. 1999:43:595-606.

22. McMurray JJ, Adamopoulos S, Anker SD, Auricchio A, Böhm M, Dickstein K, Falk V, Filippatos G, Fonseca C, Gomez-Sanchez MA, Jaarsma T, Køber L, Lip GY, Maggioni AP, Parkhomenko A, Pieske BM, Popescu BA, Rønnevik PK, Rutten FH, Schwitter J, Seferovic P, Stepinska J, Trindade PT, Voors AA, Zannad F, Zeiher A, Task Force for the Diagnosis and Treatment of Acute and Chronic Heart Failure 2012 of the European Society of Cardiology, Bax JJ, Baumgartner H, Ceconi C, Dean V, Deaton C, Fagard R, Funck-Brentano C, Hasdai D, Hoes A, Kirchhof P, Knuuti J, Kolh P, McDonagh T, Moulin C, Popescu BA, Reiner Z, Sechtem U, Sirnes PA, Tendera M, Torbicki A, Vahanian A, Windecker S, McDonagh T, Sechtem U, Bonet LA, Avraamides $P$, Ben Lamin HA, Brignole M, Coca A, Cowburn P, Dargie H, Elliott $P$, Flachskampf FA, Guida GF, Hardman S, lung B, Merkely B, Mueller C, Nanas JN, Nielsen OW, Orn S, Parissis JT, Ponikowski P, ESC Committee for Practice Guidelines. ESC guidelines for the diagnosis and treatment of acute and chronic heart failure 2012: The Task Force for the Diagnosis and Treatment of Acute and Chronic Heart Failure 2012 of the European Society of Cardiology. Developed in collaboration with the Heart Failure Association (HFA) of the ESC. Eur J Heart Fail. 2012;14:803-69.

23. Contini M, Apostolo A, Cattadori G, Paolillo S, lorio A, Bertella E, Salvioni E, Alimento M, Farina S, Palermo P, Loguercio M, Mantegazza V, Karsten M, Sciomer S, Magrì D, Fiorentini C, Agostoni P. Multiparametric comparison of CARvedilol, vs. NEbivolol, vs. Blsoprolol in moderate heart failure: the CARNEBI trial. Int J Cardiol. 2013;168:2134-40.

24. Poole-Wilson PA, Swedberg K, Cleland JG, Di Lenarda A, Hanrath P, Komajda M, Lubsen J, Lutiger B, Metra M, Remme WJ, Torp-Pedersen C, Scherhag A, Skene A, Carvedilol Or Metoprolol European Trial Investigators. Comparison of carvedilol and metoprolol on clinical outcomes in patients with chronic heart failure in the Carvedilol Or Metoprolol European Trial (COMET): randomised controlled trial. Lancet. 2003:362:7-13.

25. Dogan A, Karabacak M, Tayyar S, Erdogan D, Ozaydin M. Comparison of the effects of carvedilol and nebivolol on diastolic functions of the left ventricle in patients with non-ischemic heart failure. Cardiol J. 2014;21:76-82. 